ISSN 2073-4360

www.mdpi.com/journal/polymers

Article

\title{
Spontaneous Vesicles Modulated by Polymers
}

\section{Mercedes Velázquez ${ }^{1, *}$, Margarita Valero ${ }^{1}$ and Francisco Ortega ${ }^{2}$}

1 Departamento de Química Física, Universidad de Salamanca, E-37008 Salamanca, Spain; E-Mail: mvalero@usal.es

2 Departamento de Química Física I, Facultad de Ciencias Químicas, Universidad Complutense de Madrid, E-28040 Madrid, Spain; E-Mail: fortega@quim.ucm.es

* Author to whom correspondence should be addressed; E-Mail: mvsal@usal.es.

Received: 7 June 2011; in revised form: 19 July 2011 / Accepted: 4 August 2011 /

Published: 8 August 2011

\begin{abstract}
Vesicles are widely used in technological applications including cosmetic products, in microencapsulation for drug delivery, as anticancer agents and in the technology of adhesives, paints and inks. The vesicle size and the surface charge are very important properties from a technological point of view. Thus, the challenge in formulation is to find inexpensive stable vesicles with well-defined sizes and to modulate the surface charge of these aggregates. In this work we analyze the effect of different polymers on the structural properties of vesicles of the biodegradable surfactant sodium bis(2-ethyl-hexyl) sulfosuccinate, Aerosol OT. Using fluorescence, conductivity, electrophoretic mobility and dynamic light scattering measurements we study the effect of the polymer nature, molecular weight and polymer concentration on the stability and the vesicle size properties. Results demonstrate that it is possible to modulate both the size and the electric surface charge of spontaneous vesicles of Aerosol OT by the addition of very small percentages of poly(allylamine) and poly(maleic anhydride-alt-1-octadecen).
\end{abstract}

Keywords: Aerosol OT; light scattering measurements; $\zeta$-potential; electrical conductivity; poly(maleic anhydride-alt-1-octadecen); poly(allylamine) 


\section{Introduction}

Vesicles consist of a surfactant bilayer that separates an inner region of water from a continuous phase of the same fluid. The majority of processing methods for unilamellar vesicles production use an input of energy, ultrasonic treatment, elevated temperature or pressure [1] to disperse surfactants as a vesicle phase that in most cases is unstable and highly polydisperse. However, spontaneous formation of vesicles has been reported either by mixing surfactants with oppositely charged head groups [2-6] or with double-tailed surfactants [7-9] and with block-copolymer systems [10-13].

Taking into account that vesicles are widely used in technological applications including cosmetic products, microencapsulation for drug delivery [14], as anticancer agents [15] or in the technology of adhesives, paints, inks [16], the challenge is to find inexpensive stable vesicles with well-defined properties. Theoretical approaches have been developed to determine the optimal characteristics of a vesicle. These models show that small variations in composition are enough to result in large change in the optimal size or in the stability of vesicles [17-20].

It is well known that vesicles can be tuned with polymers [21-26]. Thus, the inclusion of small percentages of polymers in liposome formulation used as drug carriers increases their circulation in vivo, favoring the uptake in the target site [27]. However, a rigorous control of the polymer concentration and of the polymer mass becomes necessary, because polymer-lipid liposomes tend to form micelles when the polymer molecules exceed a certain concentration [28]. In order to gain insights into the effect of the polymer nature and concentration on vesicle properties, we have studied the stability and properties of vesicles of Aerosol OT, Scheme 1, mixed with different water soluble polymers [8,9,29]. We chose this surfactant because it is biodegradable [30] and it forms spontaneous unilamellar vesicles in water with a critical vesicle concentration, cvc, of $(7.5 \pm 0.3) \mathrm{mM}$ at $29.9{ }^{\circ} \mathrm{C}[8,31]$. The polymers used in these works were poly(ethylene) glycol, PEG $17 \mathrm{~K},(17 \mathrm{kDa})$ and poly(sodium4-styrensulfonate), PSS, [8,9]. For comparative purposes we also studied the effect of $\mathrm{NaCl}$ on the vesicle properties $[8,9]$. Results showed that the addition of PSS, PEG K 17 and $\mathrm{NaCl}$ favors the formation of spontaneous vesicles of Aerosol OT. However, the effect induced by additives is different; thus, in the case of PSS the stabilization is due to the screening of the surface charge by the polymer counterions, $\mathrm{Na}^{+}$. The effect is almost similar to the addition of $\mathrm{NaCl}$. In contrast, the addition of PEG 17K stabilizes the Aerosol OT vesicles by the adsorption of the polymer in the vesicle interface [9].

All these works were carried out with dilute polymer solutions. However, it is also important to investigate the effect of both, the polymer concentration and the polymer mass on the stability of vesicles. Therefore, we expanded the polymer concentration at the semidilute regime and the polymer mass from $6 \mathrm{kDa}$ (PEG 6K) to $35 \mathrm{kDa}$, PEG 35K [29]. Results showed that the PEG-surfactant mixed vesicles are stable in a narrow polymer concentration range. Even in dilute polymer solutions, different kind of aggregates appear and finally, they are turned into polymer-surfactant mixed micelles [29]. The vesicle-micelle transition was interpreted in terms of the packing parameter [29] and it takes place via intermediate structures such as elongated micelles. Results point to the formation of PEG layers at the vesicle interface as the driving-force for the vesicle $\rightarrow$ micelle transition [29]. 
Scheme 1. Molecular structures of the surfactant Aerosol OT and the polymers: poly(maleic anhydride-alt-1-octadecen) (PMAO) and poly(allylamine) (PA).

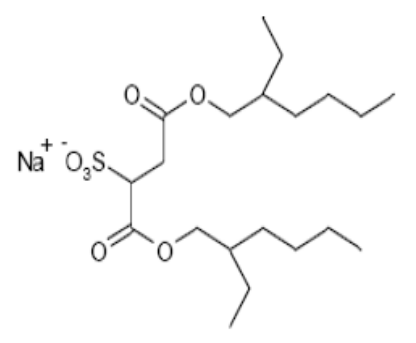

Aerosol OT

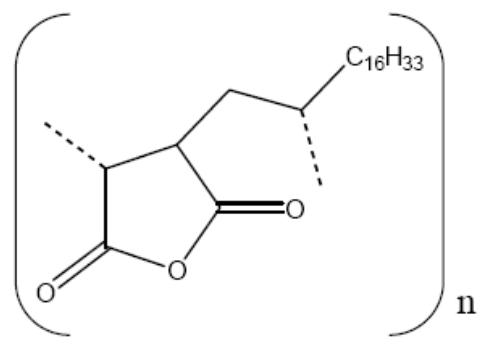

PMAO

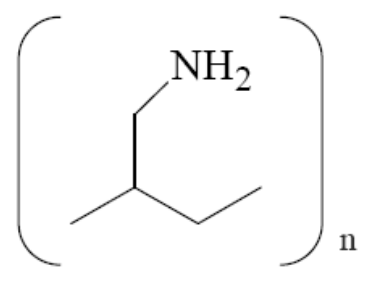

PA

To systematically investigate the effect of polymers on the vesicle properties, it is necessary to work with oppositely charged polymer and surfactant molecules and with a water insoluble polymer. With this objective in mind we have selected the following polymers, poly(allylamine) (PA) and poly(maleic anhydride-alt-1-octadecen) (PMAO), Scheme 1. The polyelectrolyte poly(allylamine) (PA) is in the cationic form at the water $\mathrm{pH}(\mathrm{pKa}=9.7)$ [32], consequently, it can interact with the sulphonate groups of the surfactant Aerosol OT [33]; therefore, it offers the possibility of investigating the effect of the oppositely charged polymers on the vesicle properties. In contrast, the polymer poly(maleic anhydride-alt-1-octadecen) (PMAO) is a water insoluble molecule which forms stable Langmuir monolayers [34] at the air-water interface; consequently, it is solubilized in the vesicle bilayer, thereby it offers the possibility of studying the effect of molecules incorporated into the membrane bilayer on the vesicle structure and properties. To accomplish these objectives we obtain the cve of mixed aggregates with different polymer composition by using electrical conductivity measurements. On the other hand, we use dynamic light scattering and electrophoretic mobility measurements to study the effect of the addition of PMAO and PA on the size of vesicles and on the electric properties of the vesicles surface, respectively. 


\section{Experimental Section}

\subsection{Reagents and Vesicle Preparation}

The surfactant sodium bis(2-ethyl-hexyl) sulfosuccinate, named Aerosol OT or AOT, was from Fluka. We use the purification method published elsewhere [35] and the surfactant purity was evaluated using gas chromatography [36]. The polymer poly(maleic anhydride-alt-1-octadecen), PMAO $\left(\mathrm{M}_{\mathrm{r}}=40 \mathrm{kDa}\right)$ was from Sigma-Aldrich and was used as received without further purification. The aqueous solution of poly(allylamine) $20 \% \mathrm{w}: \mathrm{w}, \mathrm{PA}\left(\mathrm{M}_{\mathrm{r}}=17 \mathrm{kDa}\right)$ was from Sigma-Aldrich. The polymer molecular weights were provided by the manufacturer.

To obtain spontaneous aggregates, the surfactant solutions were prepared without external energy input. The AOT/PA vesicles were prepared by dissolving the surfactant in the aqueous PA solutions with a given polymer concentration. We use two different procedures for systems containing AOT and PMAO because PMAO is a water-insoluble molecule. In the first one, the aggregates were prepared by weight both, the surfactant and polymer molecules, and dissolving them in water. In the second procedure the polymer PMAO was dissolved in surfactant solutions with a given concentration. DLS measurements show that the size of the aggregates prepared by the two different methods agrees with one another. The solutions were prepared with water purified using the combination of RiOs and Milli-Q systems from Millipore.

To study the effect of polymer concentration on the properties of Aerosol OT vesicles, the aggregates were prepared with $0.03 \mathrm{M}$ of Aerosol OT and different polymer concentration. The maximum polymer concentrations used were $0.020 \%$ for PA and $0.094 \%$ for PMAO, because the systems become instable above these polymer concentrations. In the case of PMAO/Aerosol OT mixtures, above $0.1 \%$ of PMAO the polymer molecules precipitate, probably because vesicles cannot incorporate more PMAO inside their bilayers. On the other hand, large flocs are clearly observed in vesicles prepared with $(\mathrm{PA})>0.02 \%$, the non-uniform adsorption of PA on the vesicle surface can interpret this behavior, see below. All solutions were prepared the day before to obtain experimental measurements and were maintained at $30^{\circ} \mathrm{C}$. The measurements were carried out with clear solutions.

\subsection{Conductivity Measurements}

The electrical conductivity was measured with a conductometer model 727 from Metrohm operated at $2.4 \mathrm{kHz}$. A Metrohm Herisau conductivity cell, model AG 9101, was used. The cell constant, $0.847 \mathrm{~cm}^{-1}$, was obtained by calibration with $\mathrm{KCl}$ solutions of known concentrations [37]. Because the correct determination of the cvc requires a large number of experimental data, a conductometric titration was employed. During titration, solutions obtained by successive dilutions were allowed to equilibrate a few minutes until a stable measurement was obtained [38,39].

\subsection{Light Scattering Measurements (DLS)}

Dynamic light scattering was performed with a CGS-8 from ALV GmbH working in pseudo cross-correlation mode, using the green line $(\lambda=514.5 \mathrm{~nm})$ of an argon ion laser (Coherent I300). The intensity correlation functions were obtained at a fixed temperature of $30.0^{\circ} \mathrm{C}$ and as a function of the 
scattering angle, $\theta$, between 30 and $140^{\circ}$, corresponding to wavevectors, $\mathrm{q}$, from $8.42 \times 10^{4}$ to $3.14 \times 10^{5} \mathrm{~cm}^{-1}$ defined as: $\mathrm{q}=\frac{4 \pi \mathrm{n}}{\lambda} \sin \left(\frac{\theta}{2}\right)$; where $n$ represents the solution refractive index.

The normalized second order correlation functions $\left(g^{(2)}(t)\right)$ were analyzed using both GENDIST [40] and CONTIN [41,42] inverse Laplace algorithms, giving both similar relaxation time distributions. The solution chosen was the most probable one and corresponds with a value of the regularization parameter, $\alpha=0.5$. From the average relaxation times, $\tau$, the apparent diffusion coefficients, $\mathrm{D}^{\text {app }}$, were obtained and using the Stokes-Einstein relation, the apparent hydrodynamic radii were calculated [43].

$$
1 / \tau=D^{\text {app }} q^{2}=\frac{k_{B} T}{6 \pi \eta R_{H}^{\text {app }}} q^{2}
$$

\subsection{Zeta potential Measurements}

The electrophoretic mobility measurements were carried out by means of the laser Doppler electrophoresis technique using the Zetasizer 3000 device (Malvern, UK). All experiments were made in a $5 \mathrm{~mm} \times 2 \mathrm{~mm}$ rectangular quartz capillary. Each experimental value is the average of ten measurements and the standard deviation of these measurements was considered the experimental error. The electrophoretic mobility values were also obtained at $30.0^{\circ} \mathrm{C}$. The electrophoretic mobility, $\mu_{\mathrm{e}}$, was converted into zeta-potential, $\zeta$, using the Smoluchowski's relation, $\zeta=\frac{\mu_{e} \eta}{\varepsilon}$ where $\eta$ and $\varepsilon$ are the viscosity and the permittivity of the solvent, respectively.

\section{Results and Discussion}

\subsection{Electrical Conductivity Measurements}

In previous works we used the 4-[6-methoxy-2-napthyl]-2-butanone, nabumetone, as fluorescent probe to obtain the critical vesicle concentrations of Aerosol OT vesicles [9,29]. Even though this probe has been successfully used to study different kinds of surfactant aggregation processes [9,29,44,45], it was not used in our systems because the PA and PMAO polymers present fluorescence emission. Therefore, we use electrical conductivity measurements to obtain the critical vesicle concentration.

Figure 1 shows the experimental conductivity values as a function of the Aerosol OT for aqueous solutions of mixed polymer-Aerosol OT with polymer concentration constant. The vesicle critical concentration ( $\mathrm{crc}$ ) is assigned with the break point of the experimental data [8]. For the sake of clarity, the figure presents results for some polymer-surfactant systems, a similar trend was observed for the rest of mixtures.

The results show two different trends as a function of the polymer added to the Aerosol OT vesicles. Thus, the cvc values of mixed PMAO-Aerosol OT vesicles are independent of polymer concentration and the average value found is $(7.0 \pm 0.5) \mathrm{mM}$. This value is quite similar to the cvc of pure Aerosol OT vesicles [8]. In contrast, the cve values obtained for PA-Aerosol OT mixed vesicles are lower than the value found for pure Aerosol OT and they are almost independent of the polymer concentration. The cvc average value calculated was $(2.7 \pm 0.5) \mathrm{mM}$. This is the classical behavior of strongly interacting polymer-surfactant mixtures [46]. It is also interesting to note that the cve of 
PA-Aerosol OT mixed vesicles is in the order of magnitude of the cvc obtained previously for stable mixed vesicles of PEG/AOT or PSS/AOT $[8,9]$.

Figure 1. Electrical conductivity values of surfactant solutions: (triangles) sodium bis(2-ethyl-hexyl) sulfosuccinate (Aerosol OT or AOT) dissolved in $0.0001 \%$ of poly(allylamine) (PA), (circles) AOT dissolved in 0.02\% PA and (squares) AOT dissolved in $0.094 \%$ poly(maleic anhydride-alt-1-octadecen) (PMAO).

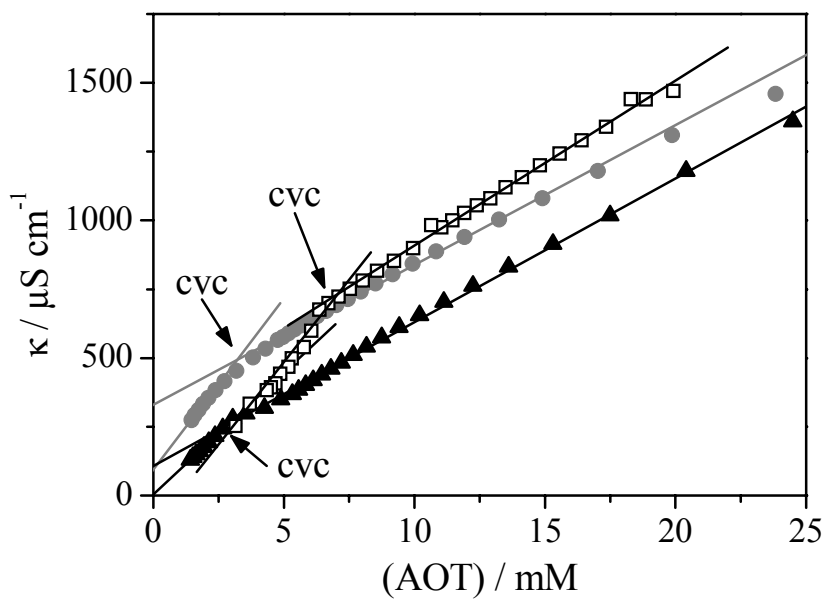

From the results it is possible to conclude that the inclusion of PMAO on the vesicle bilayer does not affect the critical vesicle concentration of Aerosol OT, while a significant lowering of the cve is observed when vesicles are prepared with oppositely charged polymer and surfactant molecules. This means that the surfactant Aerosol OT molecules prefer to aggregate with the polymer PA than themselves [46].

\subsection{Light-Scattering Measurements}

In order to obtain information about the effect of polymer addition on the aggregates size, dynamic light-scattering experiments have been performed at $30.0{ }^{\circ} \mathrm{C}$ with different polymer concentrations. The Aerosol OT concentration was kept constant at $0.03 \mathrm{M}$. The hydrodynamic radius of pure Aerosol OT vesicle, $\mathrm{R}_{\mathrm{H}}^{\mathrm{app}}=(69 \pm 2) \mathrm{nm}$, was previously determined using dynamic light scattering measurements [29] and it is independent of the surfactant concentration for [AOT] $\leq 0.03 \mathrm{M}$.

The correlation functions for AOT/PMAO systems are monomodal and the inverse time decay shows a linear dependence with $\mathrm{q}^{2}$, Figure 2(a). The apparent hydrodynamic radius was calculated from the apparent diffusion coefficient obtained from the slope of inverse time decay $v$ s. $\mathrm{q}^{2}$ and the Stokes-Einstein equation. The apparent hydrodynamic radius is represented against the polymer concentration in the Figure 2(b). Examination of results in the Figure 2(b) shows that the hydrodynamic radius is almost independent of polymer concentration at low polymer concentrations with a size quite similar to the Aerosol OT vesicles. This fact seems to indicate that the incorporation of small concentrations of PMAO inside the vesicles bilayer does not affect the size of Aerosol OT vesicles. However, when the polymer concentration incorporates onto the bilayer is further increased the apparent hydrodynamic radius increases from 70 to $118 \mathrm{~nm}$. 
Figure 2. (a) The inverse decay time values vs. the square wavevector for mixtures of Aerosol OT $0.03 \mathrm{M}$ with PMAO $0.0824 \%$ w:w. (b) Variation of the apparent hydrodynamic radius of vesicles with PMAO concentration. The open circle represented on the PMAO concentration of $10^{-4} \%$ corresponds to the hydrodynamic radius of Aerosol OT vesicles. The Aerosol OT concentration was kept constant at $0.03 \mathrm{M}$.
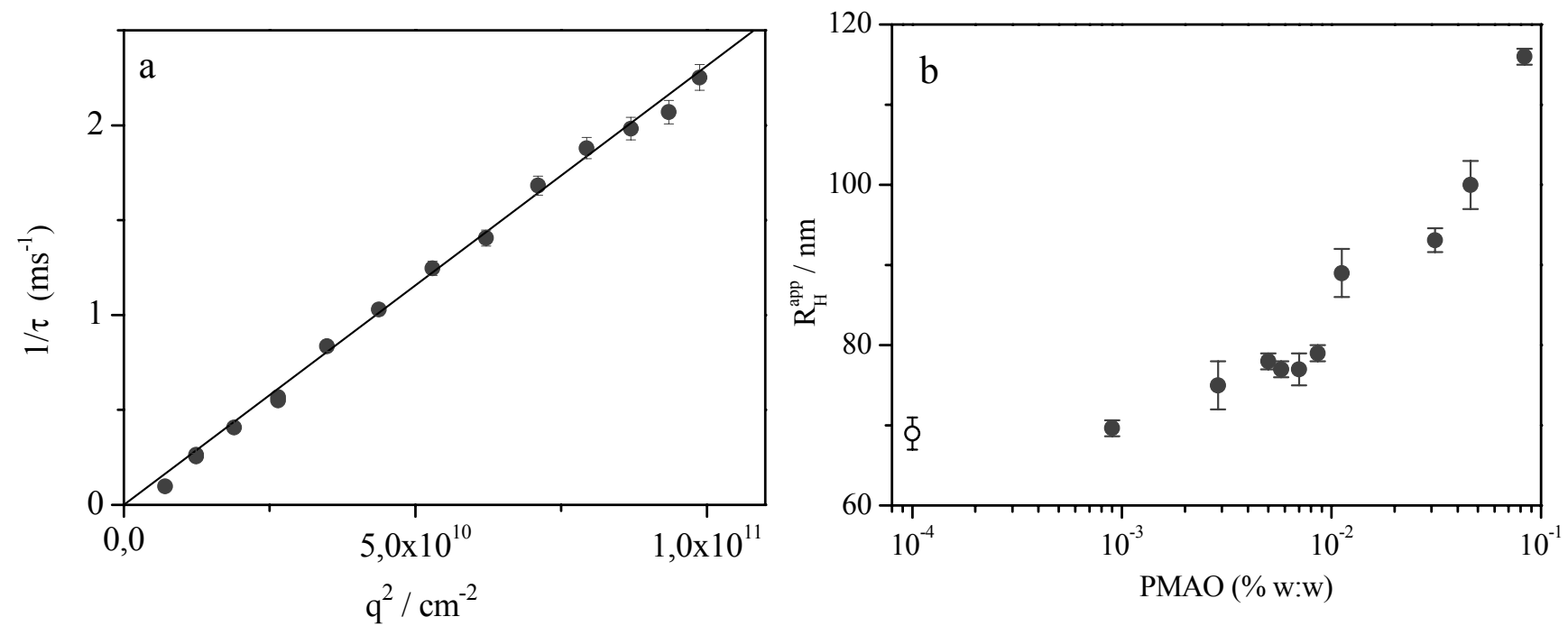

All the experiments corresponding to mixtures of Aerosol OT with PA present single exponential correlation functions that, when analyzed using regularized Inverse Laplace Transforms (ILT) (GENDIST, CONTIN), lead to single peak decay time distributions. The inverse time decay shows the expected linear dependence with $\mathrm{q}^{2}$. From the linear dependence of the inverse decay time with $\mathrm{q}^{2}$ we calculate the apparent diffusion coefficient and using the Stokes-Einstein relation we found the apparent hydrodynamic radius. The $\mathrm{R}_{\mathrm{H}}{ }^{\text {app }}$ values are represented against PA concentration in the Figure 3 .

Figure 3. Changes on the apparent hydrodynamic radius values with the polymer concentration for vesicles prepared with Aerosol OT $0.03 \mathrm{M}$ and different PA concentration. The open circle represented on $(\mathrm{PA})=10^{-6} \%$ corresponds to the hydrodynamic radius of Aerosol OT vesicles.

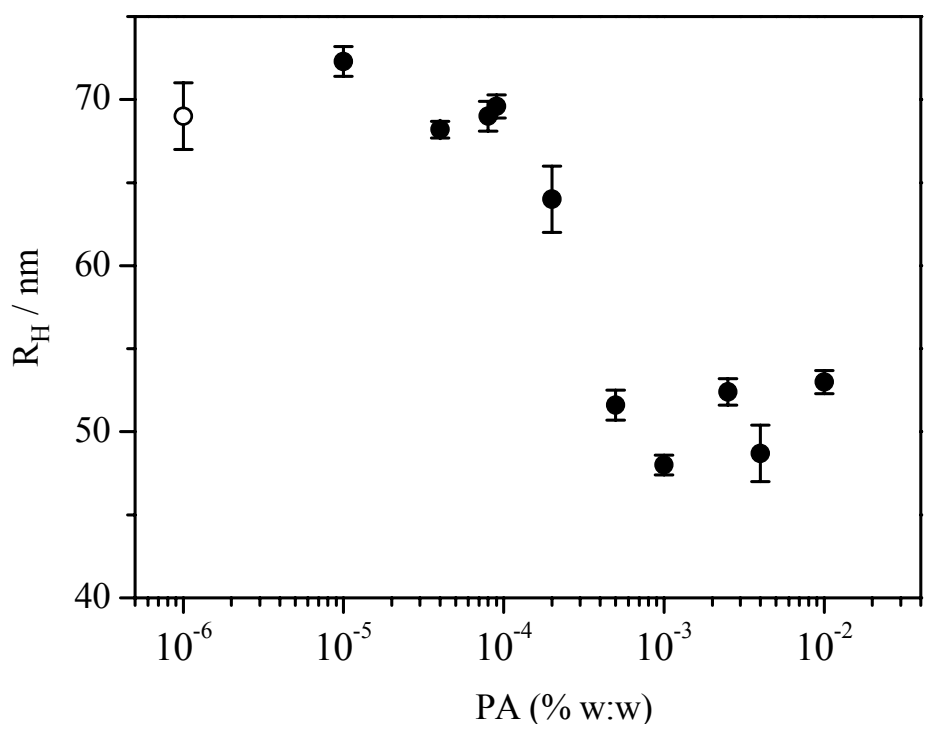


Examination of Figure 3 shows that for relatively small polymer concentrations, [PA] $<1 \times 10^{-4} \%$ w:w, vesicles have the same size than the Aerosol OT ones and when PA concentration increases up to this value, the hydrodynamic radius decreases with PA concentration until it reaches a constant value of $(50 \pm 1) \mathrm{nm}$ at the polymer concentration of $4 \times 10^{-4} \%$.

It was not possible to obtain information about the morphology of the different aggregates by means of Cryo-TEM, because no clear images were obtained in these systems. This fact was previously observed in mixtures of Aerosol OT with PEG polymers [29] and it was attributed to association processes between vesicles in the freezing procedure $[47,48]$.

\subsection{Electrophoretic Mobility Results}

The effect of the polymer addition on the zeta potential of Aerosol OT vesicles was also investigated. Figure 4 presents the $\zeta$-potential values of vesicles prepared with $0.03 \mathrm{M}$ of Aerosol OT and different PMAO and PA concentrations. For comparison, Figure 4 also presents the $\zeta$-potential of $0.03 \mathrm{M}$ Aerosol OT vesicles, shown by the open circle in the figure.

Results in Figure 4 show that in mixtures containing low PMAO concentrations, the $\zeta$-potential remains constant in the value of pure Aerosol OT vesicles. This fact indicates that in this polymer concentration range, mixed vesicles are quite similar to the pure Aerosol OT ones. When the PMAO concentration is further increased the $\zeta$-potential weakly decreases. This fact agrees very well with results obtained by other authors $[49,50]$. In these works, the combination of light scattering and $\zeta$-potential measurements indicated that the zeta-potential often decreases with increasing the vesicle radius.

Figure 4. $\zeta$-potential values for vesicles prepared with $0.03 \mathrm{M}$ Aerosol OT and different polymer concentrations. Solid circles represent results for PA/Aerosol OT vesicles and triangles for PMAO/Aerosol OT mixtures. The $\zeta$-potential of Aerosol OT vesicles is represented as the open circle. The dotted line shows the limit $\zeta$-potential value for stable vesicles, see text. The solid line is a visual guide of the tendency.

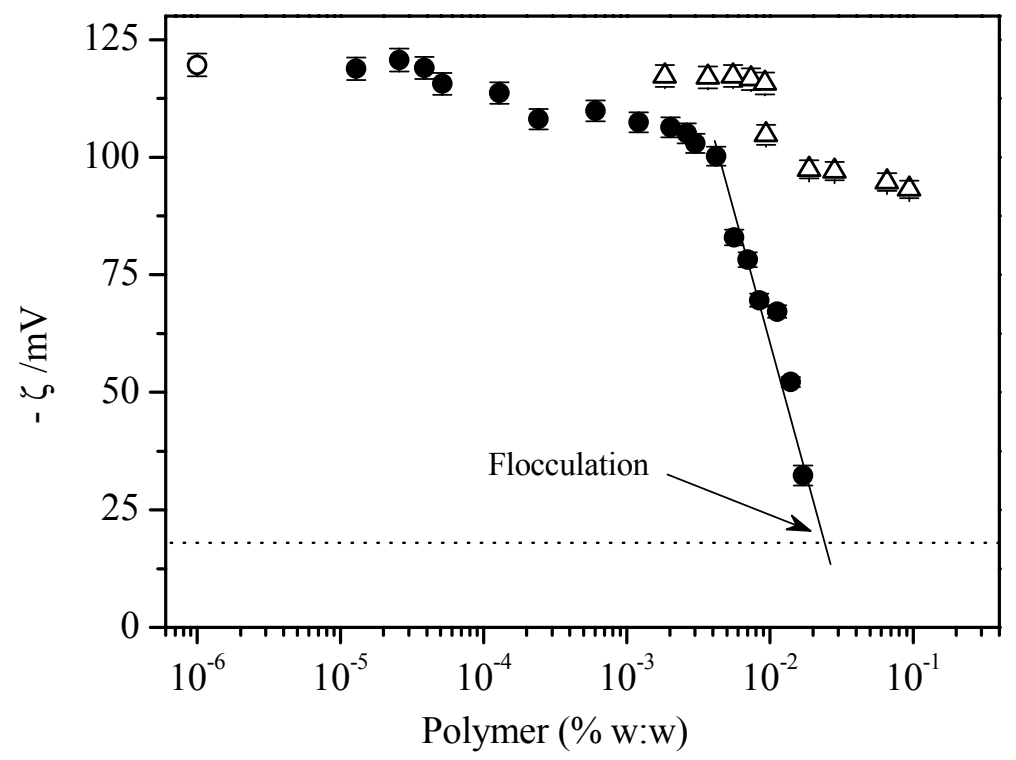


The $\zeta$-potential values for PA/Aerosol OT vesicles are almost independent of polymer concentration until the polymer concentration reaches a value of $0.005 \% \mathrm{w}: \mathrm{w}$. Above this polymer concentration, the $\zeta$-potential of vesicles sharply decreases indicating that the polycation PA partially neutralizes the surface negative charge of the Aerosol OT vesicles.

It was not possible to obtain the isoelectric conditions, because when the polymer concentration reaches a value above $0.02 \%$ large flocs are observed. It is interesting to note that, at this polymer concentration, the vesicle electric charge is not completely neutralized. This phenomenon cannot be interpreted by the framework of DLVO theory, because in this theory the particle surface is assumed to be uniformly charged [51]; however, this assumption could not be fulfilled in the case of polyelectrolyte adsorption onto the surface of particles. It is well established that the adsorption of polyelectrolytes on the oppositely charged surface takes place in a highly correlated manner resulting in a non-uniform distribution of the adsorbed chains and, consequently, of the surface electric charge. Volegol and Twar recently developed a model for the potential of mean force between non-uniformed charged particles. They showed that, the inter-particle potential has an attractive component even in particles bearing an electric charge of the same sign. This potential is responsible of the evolution from the initial monodisperse vesicles toward a broader distribution of large aggregates that finally flocculate [52]. According to this model the relation between the $\zeta$-potential and the radius of stable aggregates $(\mathrm{R})$ is given by [53]:

$$
R=\frac{10 k_{B} T}{\pi \varepsilon \ln 4(\zeta)^{2}}
$$

where $\mathrm{k}_{\mathrm{B}}$ represents the Boltzmann's constant and $\varepsilon$ is the permittivity of water. Taking into account that the radius found in this work for stable Aerosol OT/PA mixed vesicles is $50 \mathrm{~nm}$, we use the Equation (1) to estimate the $\zeta$-potential of vesicles with this radius. The $\zeta$-potential value estimated from Equation (1) is $\pm 17 \mathrm{mV}$. According to the Volegol's model vesicles with $\zeta$-potential values above $|17| \mathrm{mV}$ are stable. Our results are consistent with this model because the $\zeta$-potential values measured for Aerosol OT/PA vesicles prior flocculation were higher, in absolute value, than $17 \mathrm{mV}$. On the other hand, from results in the Figure 4 we can estimate the polymer concentration needed to reach the $\zeta$-potential corresponding to unstable Aerosol OT/PA vesicles $(-17 \mathrm{mV})$. The estimated value was $0.025 \%$, in good agreement with the phase separation observed for PA concentrations above $0.02 \%$.

\section{Conclusions}

We have studied the effect of different type of polymers on the properties of Aerosol OT vesicles by using electric conductivity, dynamic light scattering and $\xi$-potential measurements. Results obtained in this work show that the properties of polymer/Aerosol OT vesicles significantly depend on the polymer nature. We interpret this behavior on the basis of the different polymer-surfactant interactions. Thus, the incorporation of an insoluble polymer (PMAO) on the vesicle bilayer weakly modifies the vesicle properties, while the polymers adsorbed at the interface such as PA modify the stability and the properties of vesicles. Thus, the polycation PA is bound to the vesicle surface and partially neutralizes the negative charge of the Aerosol OT vesicles. One of the most interesting results found was that mixed 
vesicles of Aerosol OT and poly(allylamine) become unstable when the electric charge of vesicles is not completely neutralized. This behavior was interpreted using the Volegol's [50,51] model. This model considers that the non-uniform distribution on the surface electric charge due to the highly correlated adsorption of the polyelectrolyte on the vesicle surfaces induces attractive interactions between vesicles that finally flocculate even though the surface charge of vesicles is not completely neutralized. The asymmetric adsorption of PA in the inner and outer surfaces of vesicles [54] is also responsible of the decrease in the vesicle size as compare with the size of pure Aerosol OT vesicles.

Finally, results obtained in this work demonstrate that it is possible to modulate the size and the electric surface charge of spontaneous vesicles of Aerosol OT by the addition of very small percentages of polymers. This is an interesting result in view of the great potential of spontaneous vesicles in technical applications.

\section{Acknowledgements}

The authors thank financial support from ERDF and MEC (MAT 2010-19727 and MAT 2007-62666) and from Junta de Castilla y León (SA138A08). They also acknowledge the C.A.I. of Spectroscopy of the Universidad Complutense de Madrid for making available the light scattering facility. M.M. Velázquez is grateful to Universidad de Salamanca for the partial support of her stay in Madrid.

\section{References}

1. Evans, D.F.; Wennerström, H. The Colloidal Domain, 2nd ed.; Wiley-VCH: Berlin, Germany, 1999; pp. 308-310.

2. Tondre, C.; Caillet, C. Properties of the Amphiphilic Films in Mixed Cationic/Anionic Vesicles: A Comprehensive View from a Literature Analysis. Adv. Colloid Interface Sci. 2001, 93, 115-134.

3. Marques, E.; Khan, A.; Miguel, M.G.; Lindman, B. Vesicle Formation and General Phase Behavior in the Catanionic Mixture SDS-DDAB-Water. The Anionic-Rich Side. J. Phys. Chem. B 1998, 102, 6746-6758.

4. Marques, E.; Khan, A.; Miguel, M.G.; Lindman, B. Vesicle Formation and General Phase Behavior in the Catanionic Mixture SDS-DDAB-Water. The Cationic-Rich Side. J. Phys. Chem. B 1999, 103, 8353-8363.

5. Villeneuve, M.; Kaneshina, S.; Aratono, M. Effect of Equimolar Salt to Decyltrimethylammonium Decyl Sulfate on Vesicle Formation and Surface Adsorption. J. Colloid Interface Sci. 2003, 262, 227-234.

6. Hao, J.; Hoffmann, H. Self-Assembled Structures in Excess and Salt-Free Catanionic Surfactant Solutions. Curr. Opin. Colloid Interface Sci. 2004, 9, 279-293.

7. Viseu, M.I.; Velázquez, M.M.; Campos, C.S.; García-Mateos, I.; Costa, S.M.B. Structural Transition in a Bicationic Amphiphile System Studied by Light-Scattering, Conductivity and Surface Tension Measurements. Langmuir 2000, 16, 4882-4889.

8. Briz, J.I.; Velázquez, M.M. Effect of Water-Soluble Polymers on the Morphology of Aerosol OT Vesicles. J. Colloid Interface Sci. 2002, 247, 437-446. 
9. Valero, M.; Velázquez, M.M. Effect of the Addition of Water-Soluble Polymers on the Interfacial Properties of Aerosol OT Vesicles. J. Colloid Interface Sci. 2004, 278, 465-471.

10. Bryskhe, K.; Jansson, J.; Topgaard, D.; Schillen, K.; Olsson, U. Spontaneous Vesicle Formation in a Block Copolymer System. J. Phys. Chem. B 2004, 108, 9710-9719.

11. Norman, A.I.; Ho, O.L.; Lee, J.H.; Karim, A. Spontaneous Formation of Vesicles of Diblock Copolymer $\mathrm{EO}_{6} \mathrm{BO}_{11}$ in Water: A SANS Study. J. Phys. Chem. B 2006, 110, 62-67.

12. Yu, S.; Azzam, T.; Rouiller, I.; Eisenberg, A. "Breathing" Vesicles. J. Am. Chem. Soc. 2009, 131, 10557-10566.

13. Jia, L.; Cao, A.; Lévy, D.; Xu, B.; Albouy, P.-A.; Xing, X.; Bowick, M.J.; Li, M.-H. Smectic Polymer Vesicles. Soft Matter 2009, 5, 3446-3451.

14. Gregoriadis, G. Liposome Technology; CRC Press: Boca Raton, FL, USA, 1993.

15. Ceh, B.; Winterhalter, M.; Frederick, P.M.; Vallner, J.J.; Lasic, D.D. Stealth ${ }^{\circledR}$ Liposomes: From Theory to Product. Adv. Drug Delivery 1997, 24, 165-177.

16. Small, D. Handbook of Lipid Research; Plenum Press: New York, NY, USA, 1996.

17. Safran, S.A.; Pincus, P.; Andelman, D. Theory of Spontaneous Vesicle Formation in Surfactant Mixtures. Science 1990, 248, 354-356.

18. Porte, G.; Ligoure, C. Mixed Amphiphilic Bilayers: Bending Elasticity and Formation of Vesicles. J. Chem. Phys. 1995, 102, 4290-4298.

19. Szleifer, I.; Carignano, M. Tethered Polymer Layers. Adv. Chem. Phys. 1996, 94, 165-260.

20. Rovira-Bru, M.; Thompson, D.H.; Szleifer, I. Size and Structure of Spontaneously Forming Liposomes in Lipid/PEG-Lipid Mixtures. Biophys. J. 2002, 83, 2419-3439.

21. Bronich, T.K.; Solomatin, S.V.; Yaroslavov, A.A.; Eisenberg, A.; Kabanov, V.A.; Kabanov, A.V. Steric Stabilization of Negatively Charged Liposomes by Cationic Graft Copolymer. Langmuir 2000, 16, 4877-4881.

22. Volodkin, D.; Ball, V.; Scaf, P.; Voegel, J.C.; Mohwall, H. Complexation of Phosphocholine Liposomes with Polylysine. Stabilization by Surface Coverage versus Aggregation. Biochim. Biophys. Acta: Biomembranes 2007, 1768, 280-290.

23. Yaroslavov, A.A.; Sitnicova, T.A.; Raknyanskaya, A.A.; Yu, A.; Ermakov, T.V.; Burova, V.; Grinberg, Y.; Menger, F.M. Contrasting Behavior of Zwitterionic and Cationic Polymers Bound to Anionic Liposomes. Langmuir 2007, 23, 7539-7549.

24. Yaroslavov, A.A.; Raknyanskaya, A.A.; Yaroslavova, E.G.; Efimova, A.A.; Menger, F.M. Polyelectrolyte-Coated Liposomes: Stabilization of the Interfacial Complexes. Adv. Colloid Interface Sci. 2008, 142, 43-52.

25. Lin, C.-C.; Chang, C.-H.; Yang, Y.-M. Gelation of Spontaneously Catanionic Vesicles by Water Soluble Polymers. Colloids Surf. A 2009, 346, 66-74.

26. Huang, Z.-L.; Honh, J.-Y.; Chang, C.-H.; Yang, Y.-M. Gelation of Charged Catanionic Vesicles Prepared by a Semispontaneous Process. Langmuir 2010, 26, 2374-2382.

27. Blume, G.C.G. Liposomes for the Sustained Drug Release in vivo. Biochim. Biophys. Acta Biomembranes 1990, 1029, 91-97.

28. Montesano, G.; Bartucci, R.; Belsito, S.; Marsh, D.; Sportelli, L. Lipid Membrane Expansion and Micelle Formation by Polymer-Grafted Lipids: Scaling with Polymer Length Studied by Spin-Label Electron Spin Resonance. Biophys. J. 2001, 80, 1372-1383. 
29. Velázquez, M.M.; Valero, M.; Ortega, F.; Rodríguez-González, J.B. Structure and Size of Spontaneously Formed Aggregates in Aerosol OT/PEG Mixtures: Effects of Polymer Size and Composition. J. Colloid Interface Sci. 2007, 316, 762-770.

30. Burns, S.A.; Gardella, J.A., Jr. Quantitative ToF-SIMS Studies of Protein Drug Release from Biodegradable Polymer Drug Delivery Membranes. Appl. Surface Sci. 2008, 255, 1170-1173.

31. Mukerjee, P.; Mysels, K.J. Critical Micelle Concentration of Aqueous Surfactant Systems, National Standard Reference Data Series; National Bureau of Standards: Washington, DC, USA, 1971; p. 136.

32. Kioussis, D.R.; Smith, D.F.; Kofinas, P. Ammonium Perchlorate-Binding Poly(allylamine hydrochloride) Hydrogels for Wastewater Remediation. J. Appl. Polym. Sci. 2001, 80, 2073-2083.

33. Decher, G.; Schlenoff, J. Multilayer Thin Films: Sequential Assembly of Nanocomposite Materials; Wiley-VCH: Berlin, Germany, 2006.

34. López-Díaz, D.; Velázquez, M.M. Evidence of Glass Transition in Thin Films of Maleic Anhydride Derivatives: Effect of the Surfactant Coadsorption. Eur. Phys. J. E 2008, 26, 417-425.

35. Menger, F.M.; Yamada, K. Enzyme Catalysis in Water Pools. J. Am. Chem. Soc. 1979, 101, 6731-6734.

36. Datwani, S.S.; Stebe, K.J. Surface Tension of an Anionic Surfactant: Equilibrium, Dynamics, and Analysis for Aerosol-OT. Langmuir 2001, 17, 4287-4296.

37. Lind, J.E.; Zwoleni, J.J.K.; Fuoss, R.M. Calibration of Conductance Cells at $25^{\circ}$ with Aqueous Solutions of Potassium Chloride. J. Am. Chem. Soc. 1959, 81, 1557-1559.

38. García-Mateos, I.; Pérez, S.; Velázquez, M.M. Interaction between Cetyl Pyridinium Chloride and Water-Soluble Polymers in Aqueous Solutions. J. Colloid Interface Sci. 1997, 194, 356-363.

39. López-Díaz, D.; García-Mateos, I.; Velázquez, M.M. Synergism in Mixtures of Zwitterionic and Ionic Surfactants. Colloids Surf. 2005, 270, 153-162.

40. Jakes, J. Regularized Positive Exponential Sum (REPES) Program: A Way of Inverting Laplace Transform Data Obtained by Dynamic Light Scattering. Collect. Czech. Chem. Commun. 1995, 60, 1781-1797.

41. Provencher, S.W. A Constrained Regularization Method for Inverting Data Represented by Linear Algebraic or Integral Equations. Comput. Phys. Comm. 1982, 27, 213-227;

42. Provencher, S.W. CONTIN: A General Purpose Constrained Regularization Program for Inverting Noisy Linear Algebraic and Integral Equations. Comput. Phys. Comm. 1982, 27, 229-242.

43. Handbook of Chemistry and Physics, 60th ed.; CRC Press: Boca Raton, Fl, USA, 1980.

44. Valero, M.; Costa, S.M.B.; Santos, M.A. Conformations of a No-Steroidal Anti-Inflammatory Drug Nabumetone in Aqueous Environments. J. Photochem. Photobiol. A: Chem. 2000, 132, 67-74.

45. Valero, M.; Del Arco, A.; Rodríguez, L.J. New Insights into the Structure of CTAB Micelles in Presence of Cyclodextrins, Using Anti-Inflammatory Agents-Nabumetone, Naproxen as Fluorescent Probes. J. Inclusion Phenom. Macrocyclic Chem. 2002, 42, 121-130.

46. Jones, M.N. The Interaction of Sodium Dodecyl Sulfate with Polyethylene Oxide. J. Colloid Int. Sci. 1967, 23, 36-42. 
47. Menger, F.M.; Zang, H.; Caran, K.L.; Seredyuk, V.A.; Apkarian, R.P. Gemini-Induced Columnar Jointing in Vitreous Ice. Cryo-HRSEM as a Tool for Discovering New Colloidal Morphologies. J. Am. Chem. Soc. 2002, 124, 1140-1141.

48. Sleifer, I.; Gerasimov, O.V.; Thomsom, D.H. Spontaneous Liposome Formation Induced by Grafted Poly(ethylene oxide) Layers: Theoretical Prediction and Experimental Verification. Proc. Natl. Acad. Sci. 1998, 95, 1032-1037.

49. Carmona-Ribeiro, A.M.; Midmore, B.R. Surface Potential in Charged Synthetic Amphiphile Vesicles. J. Phys. Chem. 1992, 96, 3542-3547.

50. Nascimento, D.B.; Rapuano, R.; Lessa, M.M.; Carmona-Ribeiro, A.M. Counterion Effects on Properties of Cationic Vesicles. Langmuir 1998, 14, 7387-7391.

51. Verwey, E.J.W.; Overbeek, J.T.G. Theory of the Stability of Lyophobic Colloids; Elsevier: Amsterdam, The Netherlands, 1945.

52. Volegol, T.; Thwar, P.K. Analytical Model for the Effect of Surface Charge Nonuniformity on Colloidal Interactions. Langmuir 2001, 17, 7687-7693.

53. Truzzolillo, D.; Bordi, F.; Sciortino, F.; Cametti, C. Kinetic Arrest in Polyion-Induced Inhomogeneously-Charged Colloidal Particle Aggregation. 2008, arXiv:0804.0781v2. arXiv.org e-Print archive. Available online: http://arxiv.org/abs/0804.0781 (accessed on 4 August 2011).

54. Terreau, O.; Luo, L.; Eisenberg, A. Effect of Poly(acrylic acid) Block Length Distribution on Polystyrene- $b$-Poly(acrylic acid) Aggregates in Solution. 1. Vesicles. Langmuir 2003, 19, 5601-5607.

(C) 2011 by the authors; licensee MDPI, Basel, Switzerland. This article is an open access article distributed under the terms and conditions of the Creative Commons Attribution license (http://creativecommons.org/licenses/by/3.0/). 